\title{
Switching Memory Systems during Learning: Changes in Patterns of Brain Acetylcholine Release in the Hippocampus and Striatum in Rats
}

\author{
Qing Chang and Paul E. Gold \\ Department of Psychology, University of Illinois at Urbana-Champaign, Champaign, Illinois 61820
}

\begin{abstract}
This experiment measured acetylcholine ( $\mathrm{ACh}$ ) release simultaneously in the hippocampus and striatum while rats were trained in a cross maze. Consistent with past findings, rats initially showed learning on the basis of place (i.e., turning to the correct position relative to the room), but after extensive training, rats shifted to learning on the basis of response (i.e., turning to the right/left to find the food). Profiles of ACh release in the hippocampus and striatum were markedly different during training. In the hippocampus, ACh release increased by $\sim 60 \%$ at the onset of training and remained at that level of release throughout training, even after the rats began to show learning on the basis of turning rather than place. In the striatum, increases in ACh release occurred later, reaching asymptotic increases of $30-40 \%$, coincident with a transition from expressing place learning to expressing response learning. These findings suggest that the hippocampal and striatal systems both participate in learning in this task, but in a manner characterized by differential activation of the neural systems. The hippocampal system is apparently engaged first before the striatum is activated and, to the extent the hippocampus is important for place learning, promotes the use of a place solution to the maze. Later in training, although the hippocampus remains activated, the striatum is also activated in a manner that may enable the use of a response strategy to solve the maze. These findings may offer a neurobiological marker of a transition during skill learning from declarative to procedural learning.
\end{abstract}

Key words: hippocampus; striatum; learning strategies; interactions between memory systems; acetylcholine and regulation of memory; spatial versus response learning systems

\section{Introduction}

Different classes of memory appear to be processed by relatively distinct memory systems (Cohen and Squire, 1980; Gabrieli, 1998; Kesner, 1998; Willingham, 1998; Gold et al., 2001; Kim and Baxter, 2001; Packard, 2001; White and McDonald, 2002). The principal evidence comes from studies of multiple dissociations of brain structure and memory functions. However, there are also demonstrations showing that damage to one system can enhance learning mediated by another system (Matthews and Best, 1995; McDonald and White, 1995; Matthews et al., 1999; White and Wallet, 2000; Ferbinteanu and McDonald, 2001). These findings support the view that multiple memory systems at times compete with each other for control over learning.

Most studies of multiple memory systems use tasks that are dependent on one but not another neural system. One task in which the contributions of two systems can be contrasted is a cross maze. Rats are trained in a T-configuration of the maze, in which they are trained, for example, to go from the south arm to the east arm for reward. This simple task requirement can be solved using either response (egocentric) or place (allocentric) mechanisms. This task has a long history (Tolman et al., 1946, 1947 ) with detailed analyses of the impact of many variables on

Received Aug. 12, 2002; revised 0ct. 10, 2002; accepted Dec. 27, 2002.

This work was supported by United States Public Health Service research grants from the National Institute on Aging (AG 07648) and the National Institute of Neurological Disorders and Stroke (NS 32914), by the United States Department of Agriculture (00-35200-9839), and by the Alzheimer's Association.

Correspondence should be addressed to Dr. Paul E. Gold, Department of Psychology, University of Illinois at Urbana-Champaign, 603 East Daniel Street, Champaign, IL 61820. E-mail: pgold@uiuc.edu.

Copyright $\odot 2003$ Society for Neuroscience $\quad 0270-6474 / 03 / 233001-05 \$ 15.00 / 0$ learning (Restle, 1957). The solution used by each rat is revealed on a probe trial on which the rat begins from an arm $180^{\circ}$ (e.g., north) from the original start arm. The cross maze can be used to address the issue of whether rats preferentially use learning on the basis of habit (i.e., turn right to approach the goal) or on the basis of place (i.e., go to a particular part of the room to approach the goal). Recent evidence indicates quite clearly that both types of learning contribute to acquisition of this task. In particular, rats generally use place solutions early in training and response solutions later in training (Packard and McGaugh, 1996). Lidocaineinduced inactivation of the hippocampus or striatum decreases the expression of place and response solutions, respectively (Packard and McGaugh, 1996). Conversely, glutamate-induced activation of the hippocampal and striatal systems increases the expression of place and response solutions, respectively (Packard, 1999). Additionally, profiles of acetylcholine (ACh) release in the hippocampus and striatum, evident even before initial exposure to the maze, predict the preferred solution at the time rats reach criterion performance (McIntyre et al., 2003a). However, this predilection to use place or response learning does not provide a full explanation of the behavior because, after extensive training, most rats eventually select response strategies at the time of a probe trial (Packard and McGaugh, 1996; Packard, 1999). Therefore, rats switch from place to response [or from cognitive to habit (Packard, 1999)] solutions with extensive training. The issue for the present report is whether the switch in strategy used to solve the maze is reflected in changes in the profiles of ACh release in the hippocampus and striatum, used in this study to mark changes in the activation of these neural systems. 


\section{Materials and Methods}

Subjects. Six male Sprague Dawley rats (Hilltop Laboratories, Scottdale, PA), weighing $380-430 \mathrm{gm}$ at the time of surgery, were used as subjects. The rats were housed individually in translucent cages, with food and water available ad libitum until food restriction began. The rats were maintained in a $12 \mathrm{hr}$ light/dark cycle (lights on at 7:00 A.M.) throughout the experiment.

Surgery. Each rat was anesthetized with sodium pentobarbital (50-60 $\mathrm{mg} / \mathrm{kg}$, i.p.). The rats were placed in a stereotaxic apparatus with horizontal skull (Paxinos and Watson, 1986). Two plastic guide cannulas (3 mm, CMA/12; Carnegie Medicin, Stockholm, Sweden) were lowered into the hippocampus (coordinates, $5.0 \mathrm{~mm}$ posterior to bregma, 5.0 $\mathrm{mm}$ lateral and $4.2 \mathrm{~mm}$ ventral from the surface of the skull) and the lateral striatum $(0.3 \mathrm{~mm}$ posterior to bregma, $4.3 \mathrm{~mm}$ lateral and $3.7 \mathrm{~mm}$ ventral from the surface of the skull). The use of ventral (vs dorsal) hippocampal placement was based on evidence showing that, at least in terms of amygdala-dependent tasks, the ventral hippocampus appears to be important for competition during learning (Ferbinteanu and McDonald, 2001). Recent findings obtained with measures of ACh release in the ventral hippocampus are consistent with this view (McIntyre et al., 2002).

One-half of the subjects had cannulas in the left hippocampus and the right striatum, and one-half had the converse implantation. The guide cannulas were anchored in place with dental cement and skull screws. Stylets made to be flush with the cannula tips were inserted into the cannulas until the start of microdialysis procedures.

Behavioral procedures. Approximately 1 week after surgery, the rats were placed on a food-restriction schedule such that their body weights were gradually reduced to and maintained at $80 \%$. During this $7-10 \mathrm{~d}$ period, the animals were weighed and handled $(5 \mathrm{~min} / \mathrm{d})$ every day.

The training apparatus was an elevated plus-shaped cross maze with a black Plexiglas floor and clear Plexiglas walls. The maze had four identical arms (length $\times$ width $\times$ height, $45 \times 12.5 \times 15 \mathrm{~cm}$ ) containing food wells at their ends; the arms extended from a central platform $(12.5 \times$ $12.5 \times 15 \mathrm{~cm}$ ). Training was conducted in a lighted training room containing moderate-density extramaze cues (e.g., posters on the walls, door, and polygraph in a corner of the room).

On training trials, the arm facing the start arm was always blocked, creating a T-maze. Rats were trained to turn right to obtain a half piece of Frosted Cheerio (General Mills, Minneapolis, MN) located at the end of one arm. Thus, each rat could use either an allocentric spatial strategy (go to the arm in a fixed position of the room), or egocentric nonspatial strategy (turn right) to obtain the food reward.

On each trial, a rat was placed at the end of start arm, facing the center of the maze, and given up to $45 \mathrm{sec}$ to find and to eat the food. After the food was eaten or the time expired, the rat was placed in a holding cage for an interval such that the time from the start of one trial to the next was $60 \mathrm{sec}$. This was done to synchronize training with collection of microdialysis samples so that a 5 min microdialysis sample represented five trials. To avoid possible unintended influences of intramaze cues, the maze was turned $90^{\circ}$ clockwise on every trial.

A probe test was administered after each block of 20 trials. On the probe trial, the cross maze was configured as a T-maze with the start arm $180^{\circ}$ from the original start arm. Both arms at the ends of the T-maze were baited on the probe trial. If, on the probe trial, the rat turned in the direction that was correct during training, the behavior was termed a response solution. If the rat turned instead toward the arm now located in the correct place in the room, the behavior was termed a place solution.

Probe trials were administered near the middle of a 5 min interval between trials 20 and 21, 40 and 41, 60 and 61, 80 and 81, and after 100. Except for the time spent on the probe trial, each rat was kept in the holding cage for the remainder of the $5 \mathrm{~min}$. Thus, for each rat, there were five training blocks of 20 trials each (total $=100$ trials) and five probe trials. Training was completed on a single day within a single session.

Microdialysis procedures. One microdialysis probe was inserted through a guide cannula into the hippocampus, and another probe was inserted into the contralateral striatum. The dialysis probes were perfused continuously at a rate of $2.0 \mu \mathrm{l} / \mathrm{min}$ with artificial CSF (in mM: 128
$\mathrm{NaCl}, 2.5 \mathrm{KCl}, 1.3 \mathrm{CaCl}_{2}, 2.1 \mathrm{MgCl}_{2}, 0.9 \mathrm{NaH}_{2} \mathrm{PO}_{4}, 2.0 \mathrm{Na}_{2} \mathrm{HPO}_{4}, 1.0$ dextrose, adjusted to $\mathrm{pH} 7.4$ ) that contained a $100 \mathrm{~nm}$ concentration of the acetylcholinesterase inhibitor neostigmine.

To allow equilibration with brain extracellular fluid and to avoid temporary changes in extracellular neurotransmitter levels caused by acute tissue damage (Westerink and Timmerman, 1999), the first hour of dialysate was discarded. During this hour, the animals were kept in a holding cage with fresh bedding. Next, dialysate samples were collected every 5 min into small vials by an automatic refrigerated fraction collector (CMA/170; Carnegie Medicin). The first four samples were collected while rats remained undisturbed in the holding cage and comprised the samples for baseline values. After the baseline sampling period, rats were trained while samples continued to be collected every $5 \mathrm{~min}$. At the conclusion of behavioral testing, samples were sealed and stored at $-20^{\circ} \mathrm{C}$ until assay for ACh content.

After in vivo sampling was completed, each microdialysis probe was removed from the rat and placed into a $100 \mathrm{nM}$ ACh standard solution for $10-15 \mathrm{~min}$ to determine relative recovery.

ACh assay procedures. ACh content in each dialysate sample was assayed by HPLC in combination with an electrochemical detector (BAS; Bioanalytical Systems, West Lafayette, IN). The assay system included an ion-exchange microbore analytical column (BAS P/N MF-8904, $530 \times 1$ $\mathrm{mm}$ ), a microbore $\mathrm{ACh} /$ choline immobilized enzyme reactor containing acetylcholinesterase and choline oxidase (BAS P/N MF-8903, $50 \times 1$ $\mathrm{mm}$ ), an auxiliary electrode with radical flow electrochemical thin-layer cell and $13 \mathrm{~mm}$ thin-layer gasket, a wired enzyme electrode kit (a redox polymer film containing horseradish peroxidase coated on the surface of a $3 \mathrm{~mm}$ glassy carbon working electrode), and a low-dispersion injection valve with a $5 \mu$ l polyetheretherketone loop (Rheodyne model 9125087). Stable and relatively pulse-free flow was achieved with a Shimadzu (Tokyo, Japan) LC-10ADvp pump with microstep plunger.

The potential held by the working electrode was $100 \mathrm{mV}$ versus an $\mathrm{Ag} / \mathrm{AgCl}$ reference electrode. The mobile phase contained $50 \mathrm{~mm}$ $\mathrm{Na}_{2} \mathrm{HPO}_{4}, \mathrm{pH} 8.5$, and $0.5 \%$ Kathon (BAS P/N CF-2150). The flow rate was $140 \mu \mathrm{l} / \mathrm{min}$. Injection volume in this experiment was $2.5 \mu \mathrm{l}$. The detection limit was $<5 \mathrm{fmol}$. The assay was completed within $13 \mathrm{~min}$.

Histology. After training was completed, rats were deeply anesthetized with sodium pentobarbital and perfused with saline followed by $10 \%$ formalin solution. The brains were removed and placed in $10 \%$ formalin solution for 1-2 weeks before sectioning. Each brain was then frozen $\left(-20^{\circ} \mathrm{C}\right)$ and sliced $(50 \mu \mathrm{m})$ with a Leica (Nussloch, Germany) 1800 cryostat. Sections through probe sites were mounted on slides, dried, and stained with cresyl violet. Figure 1 illustrates acceptable placement locations for the probes.

\section{Results}

As shown in Figure 2, the rats learned to approach the correct arm quite rapidly. Accuracy improved from 50\% during the first 10 trials to $87 \%$ in the second 10 trials, and then reached and stayed at $>95 \%$ throughout the rest of training. The mean number of trials taken before beginning a run of 9 of 10 correct choices was $20.2 \pm 2.3$.

The performance observed on the probe trials administered after each 20 trials showed a steady change from place to response strategies (Fig. 3). The first probe trial occurred when mean performance was between 87 and 95\% correct (Fig. 2). On this probe trial, five of the six rats made place selections. With continued training through and beyond criterion performance, the rats changed their selection on the probe trials until, after 100 trials, all six rats made response selections (probe 1 vs $6 ; \chi^{2}=8.571 ; p<$ 0.005).

A key issue in this experiment was whether the profile of changes in ACh release during training was different in the hippocampus and striatum. Concentrations of ACh in baseline samples were $7.3 \pm 2.1$ and $37.3 \pm 12.3 \mathrm{fmol} / \mu \mathrm{l}$ for the hippocampus and striatum, respectively. As shown in Figure 4, release of ACh in the hippocampus increased by $\sim 60 \%$ immediately on the start of 

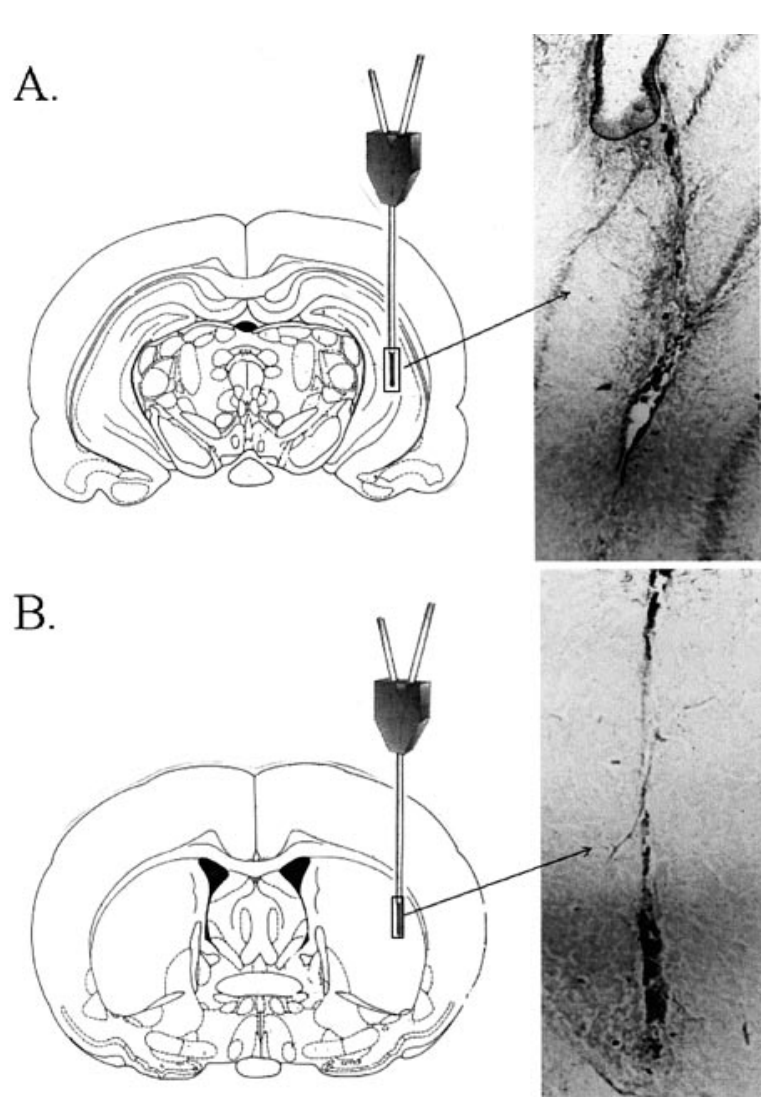

Figure 1. Examples of acceptable placements of microdialysis probes in the hippocampus ( $A$ ) and striatum (B). [Adapted from Paxinos and Watson (1986).]

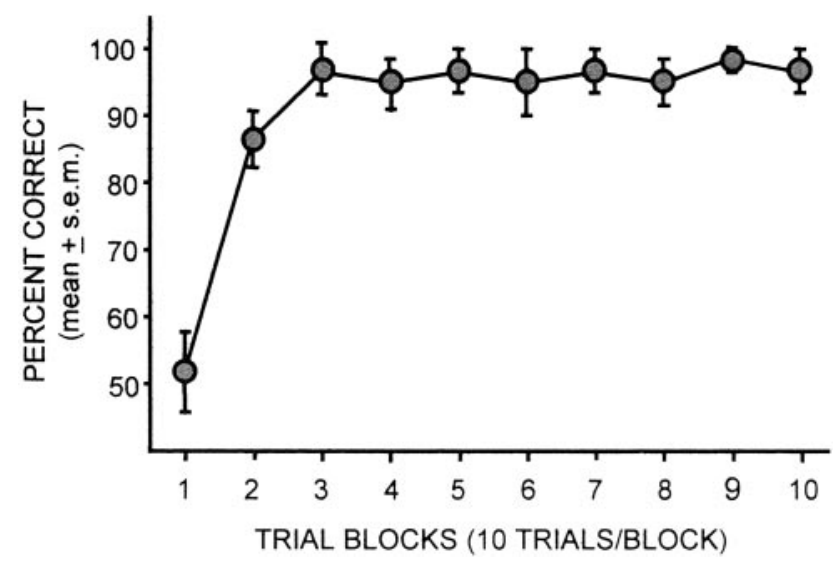

Figure 2. Rate of acquisition on cross maze. All rats reached 9 of 10 correct choices by trial 30 regardless of strategy shown on nearest probe trials.

training and remained at that level throughout the training trials. In marked contrast, $\mathrm{ACh}$ release in the striatum increased much more slowly, reaching its asymptotic increase of $30-40 \%$ after trial 25. For both hippocampal and striatal samples, the magnitudes of ACh release during the 5 min samples taken at the time of the probe trials were not different from those seen on the trials immediately before and after and are therefore not shown here.

Of particular interest, the different times at which release of ACh increased in the hippocampus versus striatum corresponded to the changes in the preferred solution displayed by rats on the probe trials. Early in training, when the rats exhibited a place strategy on probe trials, release of ACh had already in-



Figure 3. Transition from place strategy to response strategy during 100 training trials. A probe trial was administered after every 20 training trials.



Figure 4. Changes in ACh release across 100 training trials. Each sample represents $5 \mathrm{~min}$ before and during training. During training, the samples correspond to five trials per sample. $\mathrm{ACh}$ release in the hippocampus increased at the onset of training and remained stable. In contrast, $A C h$ release in the striatum grew gradually over trials as the rats switched from place to response strategy.

creased to its asymptote in the hippocampus but was still low in striatum. Later in training, when the rats switched to the use of a response strategy on the probe trial, release of ACh in the striatum had also increased. Importantly, ACh release in the hippocampus did not decline late in training but rather remained high throughout training.

In past work from our laboratory, the ratio of baseline ACh release in the hippocampus versus striatum predicted the performance on a single probe test given immediately after rats reached criterion performance (McIntyre et al., 2003a). In the present experiment, the corresponding probe trial could come between 1 and 20 trials after the criterion was reached. Although an identical measure was not available in the present experiment, it was possible to examine the relationship between baseline release of ACh and the probe test number at which the subject first used a response strategy. As shown in Figure 5, the ratio of baseline release of ACh in the hippocampus/striatum was significantly correlated with the number of trials to the first selection of a response strategy on a probe trial $(r=0.83 ; p<0.05)$. Those rats with the lowest ratio of ACh release in the hippocampus versus striatum were the first to switch from a place to a response strategy, and those with the highest ratio of ACh release in the hippocampus versus striatum were the last to switch to a response strategy. 




Figure 5. Relationship between the ratio of baseline (pretraining) release of $\mathrm{ACh}$ in the hippocampus and striatum and the probe on which the rat first switched from a place to a response solution to the maze.

\section{Discussion}

The main finding of this experiment is that the pattern of changes in ACh release in the hippocampus and striatum during learning provides a neurochemical marker of differential activation of these systems in a manner associated with differential expression of learned responses for a task that has two effective solutions. Rats quickly learned to enter one arm of the maze to obtain food. However, consistent with previous findings (Packard and McGaugh, 1996; Packard, 1999), the basis by which the rats selected the correct arm changed from place to response attributes after extended training. The present results provide evidence that increases in ACh release in the hippocampus precede increases in ACh release in the striatum during training on a task in which learning proceeds sequentially from place to response strategies. These findings provide the first neurobiological measures obtained during training marking the transition in the memory systems controlling learned performance during training.

The present findings used release of ACh to monitor the extent and timing of participation of different brain regions during learning. ACh release in the hippocampus increased to its maximum extent on the first trials and remained at that maximum throughout training, suggesting that the hippocampus was engaged at the outset of learning and remained engaged throughout extended training. In contrast, ACh release in the striatum increased more gradually, with the increase appearing as rats made their transition from selecting place solutions to selecting response solutions on probe trials. Thus, it appears that the hippocampus was activated before the striatum (i.e., at the time rats used place solutions to solve the maze). The striatal system was activated later, at the time rats began to use response solutions.

Furthermore, the present findings suggest that the hippocampus remained activated, but that the use of hippocampal processing was overridden when the striatum became fully engaged. This interpretation is consistent with the finding that inactivation of the striatum late in training leads not to chance performance but to correct performance on the basis of place learning (Packard and McGaugh, 1996). According to this view, the hippocampus retains the capacity to provide a place solution to the task and, in terms of ACh release as a marker, remains activated during performance of the task. However, this capacity to provide a place solution is obscured late in training by striatal contributions, unless the later striatal contribution is diminished or removed by pharmacological manipulation as in Packard and McGaugh
(1996). Importantly, on the basis of both measures of ACh release and manipulations of the striatum, hippocampal participation during learning does not appear to wane even after extensive training. It is intriguing to consider the possibility that the sequential activation of the hippocampal and striatal systems may reflect a transition from declarative to procedural memories.

Confirming previous findings (McIntyre et al., 2003a), the relative release of ACh in the hippocampus and striatum assessed before the beginning of training predicts which individual rats will switch from place to response solutions early and late in training. This aspect of the results suggests that individual differences in the profiles of ACh release across neural systems reflect the extent of bias between competing memory systems during learning. The present findings indicate that the bias, as well as relative release of $\mathrm{ACh}$ in the hippocampus and striatum, can change during training.

In many circumstances, training-related increases in release of acetylcholine in different neural systems provide a marker of the degree of activation of those systems during learning (Ragozzino et al., 1994, 1996, 1998; Fadda et al., 1996, 2000; Orsetti et al., 1996; Stancampiano et al., 1999; Nail-Boucherie et al., 2000; Gold et al., 2001; Hironaka et al., 2001; McIntyre et al., 2002, 2003a,b); findings within and across these studies show that locomotor activity per se does not define well the conditions when increases in ACh release are evident. These findings, like those of the present study, do not deal directly with the issue of how ACh release regulates the participation of different neural systems in learning and memory. More generally, the findings do not determine whether ACh release is an initiator or a consequence of activation. At a general level, the findings are consistent both with views applying modulation of memory formation to a systemslevel understanding of memory processing (Gold et al., 2001) and with views considering ACh to be a regulator of attention (Everitt and Robbins, 1997; Wenk, 1997). Also, although there are findings suggesting that lesions of forebrain cholinergic systems have little or no impact on learning and memory (Baxter et al., 1996; Perry et al., 2001), these findings may in part reflect limited damage to cholinergic functions (Wrenn and Wiley, 1998; Gutiérrez et al., 1999; Miranda and Bermúdez-Rattoni, 1999).

At a mechanistic level, the seemingly analogous actions of $\mathrm{ACh}$ in the hippocampus and striatum are somewhat surprising given the major differences in neuroanatomical organization of the cholinergic systems in these brain areas. Although hippocampal ACh is derived entirely from diagonal band/medial septum projection neurons, striatal ACh is primarily derived from intrinsic cholinergic neurons (Woolf and Butcher, 1981; Woolf, 1991; Calabresi et al., 2000). However, in both instances, it may be important that the axonal fields for these cells are very extensive in both the hippocampus and striatum, keeping open the possibility of similar neurobiological functions for ACh in both neural systems. For example, the extensive distribution of terminal fields seems consistent with a modulatory function for ACh released in both neural systems studied here, perhaps upregulating local processing important for these systems to contribute to performance on this task.

Using a variety of techniques, many experiments have shown that multiple memory systems are responsible for learning in the rat (McDonald and White, 1995; Gold et al., 2001; Kim and Baxter, 2001; Packard, 2001). The nature by which these systems interact is still under active exploration. The interactions at times appear to be competitive in nature; for example, the removal of the hippocampus appears to enhance learning mediated by other systems (Matthews and Best, 1995; McDonald and White, 1995; 
Matthews et al., 1999; White and Wallet, 2000; Ferbinteanu and McDonald, 2001). In past experiments using ACh release to approach this issue, there is evidence that the magnitude of $\mathrm{ACh}$ release in the hippocampus is inversely related to acquisition of a task dependent on the amygdala (McIntyre et al., 2002). However, competition is not the only form evident for interactions between memory systems. ACh release in the amygdala is positively correlated with performance on a hippocampusdependent task (McIntyre et al., 2003b). These findings suggest that the hippocampus and the amygdala have a nonreciprocal interaction in which the hippocampus competes with the amygdala but the amygdala cooperates with the hippocampus during learning. The present findings add significantly to an understanding of the relationships between neural systems important for processing different classes of learning by adding both time (the stage of training) and shifts in the relative extent of ACh release in different memory systems as features important in understanding the coordination of multiple memory systems in producing learned behavior.

\section{References}

Baxter MG, Bucci DJ, Sobel TJ, Williams MJ, Gorman LK, Gallagher M (1996) Intact spatial learning following lesions of basal forebrain cholinergic neurons. NeuroReport 7:1417-1420.

Calabresi P, Centonze D, Gubellini P, Pisani A, Bernardi G (2000) Acetylcholine-mediated modulation of striatal function. Trends Neurosci 23:120-126.

Cohen NJ, Squire LR (1980) Preserved learning and retention of patternanalyzing skill in amnesia: dissociation of knowing how and knowing that. Science 210:207-210.

Everitt BJ, Robbins TW (1997) Central cholinergic systems and cognition. Annu Rev Psychol 48:649-684.

Fadda F, Melis F, Stancampiano R (1996) Increased hippocampal acetylcholine release during a working memory task. Eur J Pharmacol 307:R1-R2.

Fadda F, Cocco S, Stancampiano R (2000) Hippocampal acetylcholine release correlates with spatial learning performance in freely moving rats. NeuroReport 11:2265-2269.

Ferbinteanu J, McDonald RJ (2001) Dorsal/ventral hippocampus, fornix, and conditioned place preference. Hippocampus 11:187-200.

Gabrieli JDE (1998) Cognitive neuroscience of human memory. Annu Rev Psychol 49:87-115.

Gold PE, McIntyre C, McNay E, Stefani MR, Korol DL (2001) Neurochemical referees of dueling memory systems. In: Memory consolidation: essays in honor of James L. McGaugh - a time to remember (Gold PE, Greenough WT, eds), pp 219-248. Washington, DC: American Psychological Association.

Gutiérrez H, Gutiérrez R, Silva-Gandarias R, Estrada J, Miranda MI, Bermúdez-Rattoni F (1999) Differential effects of 192IgG-saporin and NMDA-induced lesions into the basal forebrain on cholinergic activity and taste aversion memory formation. Brain Res 834:136-141.

Hironaka N, Tanaka K, Izaki Y, Hori K, Nomura M (2001) Memory-related acetylcholine efflux from rat prefrontal cortex and hippocampus: a microdialysis study. Brain Res 901:143-150.

Kesner RP (1998) Neurobiological views of memory. In: Neurobiology of learning and memory (Martinez Jr JL, Kesner RP, eds), pp 361-416. San Diego: Academic.

Kim JJ, Baxter MG (2001) Multiple brain-memory systems: the whole does not equal the sum of its parts. Trends Neurosci 24:324-330.

Matthews DB, Best PJ (1995) Fimbria/fornix lesions facilitate the learning of nonspatial response task. Psychon Bull Rev 2:113-116.

Matthews DB, Ilgen M, White AM, Best PJ (1999) Acute ethanol administration impairs spatial performance while facilitating nonspatial performance in rats. Neurobiol Learn Mem 72:169-179.

McDonald RJ, White NM (1995) Information acquired by the hippocampus interferes with acquisition of the amygdala-based conditioned-cue preference in the rat. Hippocampus 5:189-197.

McIntyre CK, Pal SN, Marriott LK, Gold PE (2002) Competition between memory systems: acetylcholine release in the hippocampus correlates nega- tively with good performance on an amygdala-dependent task. J Neurosci 22:1171-1176.

McIntyre CK, Marriott LK, Gold PE (2003a) Patterns of brain acetylcholine release predict individual differences in preferred learning strategies in rats. Neurobiol Learn Mem, in press.

McIntyre CK, Marriott LK, Gold PE (2003b) Cooperation between memory systems: acetylcholine release in the amygdala correlates positively with performance on a hippocampus-dependent task. Behav Neurosci, in press.

Miranda MI, Bermúdez-Rattoni F (1999) Reversible inactivation of the nucleus basalis magnocellularis induces disruption of cortical acetylcholine release and acquisition, but not retrieval, of aversive memories. Proc Natl Acad Sci USA 96:6478-6482.

Nail-Boucherie K, Dourmap N, Jaffard R, Costentin J (2000) Contextual fear conditioning is associated with an increase of acetylcholine release in the hippocampus of rat. Brain Res Cogn Brain Res 9:193-197.

Orsetti M, Casamenti F, Pepeu G (1996) Enhanced acetylcholine release in the hippocampus and cortex during acquisition of an operant behavior. Brain Res 724:89-96.

Packard MG (1999) Glutamate infused posttraining into the hippocampus or caudate-putamen differentially strengthens place and response learning. Proc Natl Acad Sci USA 96:12881-12886.

Packard MG (2001) Amygdala modulation of multiple memory systems. In: Memory consolidation: essays in honor of James L. McGaugh-a time to remember (Gold PE, Greenough WT, eds), pp 201-218. Washington, DC: American Psychological Association.

Packard MG, McGaugh JL (1996) Inactivation of hippocampus or caudate nucleus with lidocaine differentially affects expression of place and response learning. Neurobiol Learn Mem 65:65-72.

Paxinos G, Watson C (1986) The rat brain in stereotaxic coordinates, Ed 2. Orlando, FL: Academic.

Perry T, Hodges H, Gray JA (2001) Behavioural, histological and immunocytochemical consequences following 192 IgG-saporin immunolesions of the basal forebrain cholinergic system. Brain Res Bull 54:29-48.

Ragozzino ME, Wenk GL, Gold PE (1994) Glucose attenuates a morphineinduced decrease in hippocampal acetylcholine output: an in vivo microdialysis study in rats. Brain Res 655:77-82.

Ragozzino ME, Unick KE, Gold PE (1996) Hippocampal acetylcholine release during memory testing in rats: augmentation by glucose. Proc Natl Acad Sci USA 93:4693-4698.

Ragozzino ME, Pal SN, Unick K, Stefani MR, Gold PE (1998) Modulation of hippocampal acetylcholine release and spontaneous alternation scores by intrahippocampal glucose injections. J Neurosci 18:1595-1601.

Restle E (1957) Discrimination of cues in mazes: a resolution of the "placevs.-response" question. Psychol Rev 64:217-228.

Stancampiano R, Cocco S, Cugusi C, Sarais L, Fadda F (1999) Serotonin and acetylcholine release response in the rat hippocampus during a spatial memory task. Neuroscience 89:1135-1143.

Tolman EC, Ritchie BF, Kalish D (1946) Studies in spatial learning. II. Place learning versus response. J Exp Psychol 36:221-229.

Tolman EC, Ritchie BF, Kalish D (1947) Studies in spatial learning. V. Response versus place learning by the noncorrection method. J Exp Psychol 37:285-292.

Wenk GL (1997) The nucleus basalis magnocellularis cholinergic system: one hundred years of progress. Neurobiol Learn Mem 67:85-95.

Westerink BHC, Timmerman W (1999) Do neurotransmitters sampled by brain microdialysis reflect functional release? Anal Chim Acta 379:263-274.

White NM, McDonald RJ (2002) Multiple parallel memory systems in the brain of the rat. Neurobiol Learn Mem 77:125-184.

White NM, Wallet PA (2000) Dorsal hippocampal function in unreinforced spatial learning. Hippocampus 10:226-235.

Willingham DB (1998) A neuropsychological theory of motor skill learning. Psychol Rev 105:558-584.

Woolf NJ (1991) Cholinergic systems in mammalian brain and spinal cord. Prog Neurobiol 37:475-524.

Woolf NJ, Butcher LL (1981) Cholinergic neurons in the caudate-putamen complex proper are intrinsically organized: a combined Evans blue and acetylcholinesterase analysis. Brain Res Bull 7:487-507.

Wrenn CC, Wiley RG (1998) The behavioral functions of the cholinergic basal forebrain: lessons from 192 IgG-saporin. Int J Dev Neurosci 16:595-602. 